\title{
Perforación intestinal secundaria a la ingestión de múltiples imanes en un preescolar
}

\section{Intestinal perforation secondary to the ingestion of multiple magnets in a preschool child}

\author{
Iván Rivas-Rivera,* Gerardo Blanco-Rodríguez, ${ }^{\ddagger}$ Ebenezer Viridiana Cruz-Romero, ${ }^{\S}$ \\ Jaime Penchyna-Grub, ${ }^{\ddagger}$ Gustavo Teyssier-Morales ${ }^{\ddagger}$ \\ * Cirugía Pediátrica del Hospital de Gineco-Pediatría No. 31, Instituto Mexicano del Seguro Social, Mexicali, \\ Baja California Norte; ${ }^{\ddagger}$ Servicio de Cirugía de Tórax y Endoscopía del Hospital Infantil de México Federico \\ Gómez, Ciudad de México; ${ }^{\S}$ Cirugía Pediátrica del Centro Médico Naval, Ciudad de México.
}

\section{RESUMEN}

Se presentan las imágenes de radiografías y endoscopía tras la ingestión de múltiples imanes por una preescolar. Además de fotografías durante cirugía abdominal, dado que presentó perforación intestinal.

Palabras clave: Perforación intestinal, imanes, accidente, preescolar.

\section{INTRODUCCIÓN}

La ingestión de un cuerpo extraño es un accidente que se presenta con frecuencia en la edad pediátrica. ${ }^{1,2}$ En la mayoría de las ocasiones son objetos romos; sin embargo, en los últimos años, las pilas de botón y los imanes cada vez se observan con mayor frecuencia. Este tipo de objetos pueden producir daño grave que, en ocasiones, requieren de manejo quirúrgico como en el caso que nos ocupa. ${ }^{1-3}$

Los imanes compuestos de neodimio, boro y hierro son más peligrosos, dado que son 10 veces más potentes que los imanes convencionales. ${ }^{4,5}$ Cuando ocurre ingestión de múltiples imanes, pueden separarse unos de otros y atraerse entre dos asas intestinales, lo que genera presión intensa, provocando isquemia

\begin{abstract}
$X$-ray and endoscopy images are presented after the ingestion of multiple magnets by a female preschooler, as well as photographs during abdominal surgery, since she had intestinal perforation.
\end{abstract}

Keywords: Intestinal perforation, magnets, accident, preschool.

y, subsecuentemente, obstrucción, perforación, fístulas, vólvulos, infección, resección intestinal y hasta la muerte. ${ }^{3-7}$

Presentamos el caso de una niña en edad preescolar que ingirió múltiples imanes, y que en menos de 28 horas presentó una lesión intestinal.

\section{CASO CLÍNICO}

Se trata de paciente femenino de tres años y 11 meses, sin antecedentes de importancia, que refiere ingestión accidental de 17 imanes 28 horas antes del inicio de los síntomas. Presenta disfagia y odinofagia, se toma radiografía simple de tórax que muestra tres imanes localizados en faringe (Figura 1). En la placa de abdomen se observan

Correspondencia: Gerardo Blanco-Rodríguez, E-mail: drgerardoblanco@gmail.com

Citar como: Rivas-Rivera I, Blanco-Rodríguez G, Cruz-Romero EV, Penchyna-Grub J, Teyssier-Morales G. Perforación intestinal secundaria a la ingestión de múltiples imanes en un preescolar. Rev Mex Pediatr. 2021; 88(3): 116-119. https://dx.doi.org/10.35366/102193 

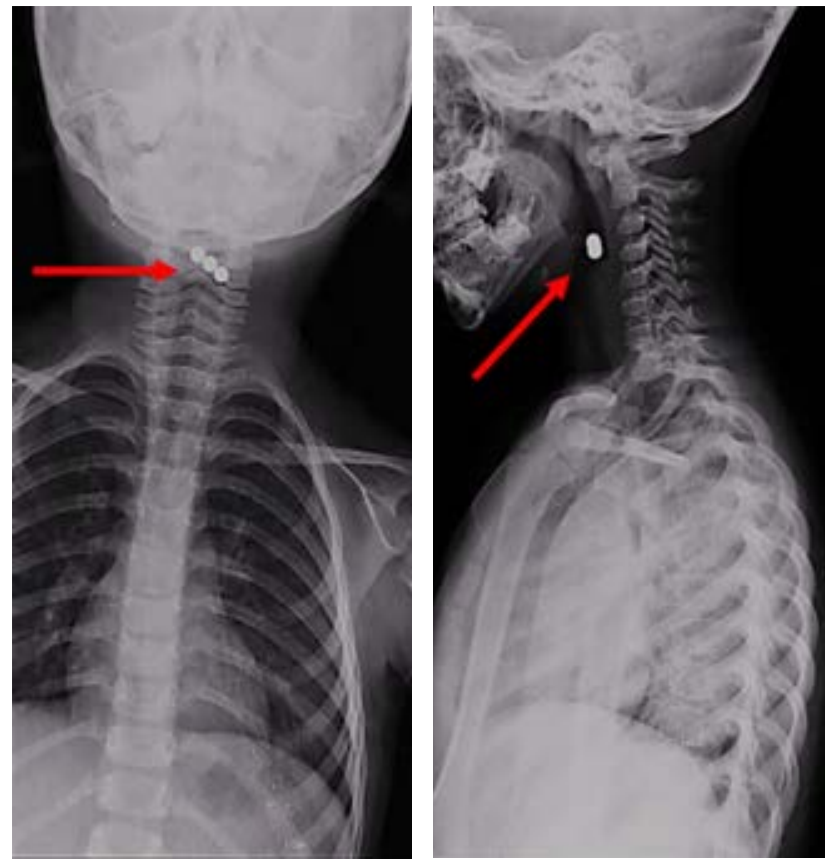

Figura 1: Radiografía anteroposterior y lateral en las que se observan tres imanes en faringe.

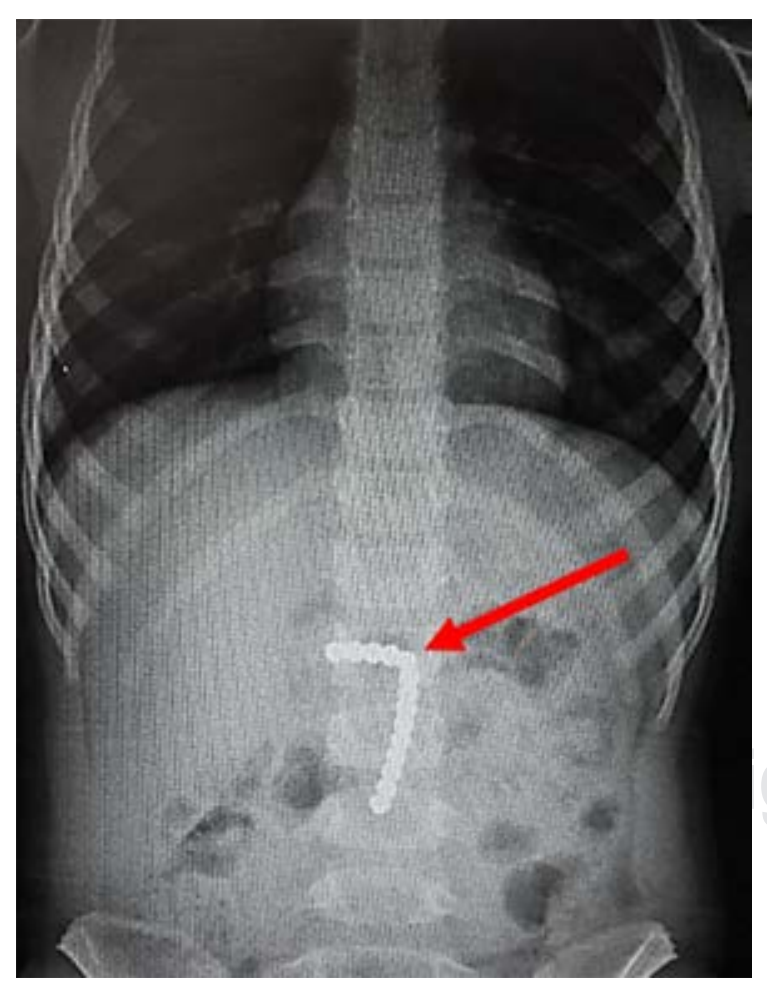

Figura 2: Radiografía anteroposterior toracoabdominal en la que se observan 14 imanes en región abdominal. los 14 imanes restantes (Figura 2). Fue enviada a nuestro hospital para ser evaluada por un servicio de Endoscopía.

A la exploración física inicial, la frecuencia cardiaca, frecuencia respiratoria, temperatura y tensión arterial eran normales para su edad. Se encontraba consciente, hidratada, ruidos cardiacos rítmicos, adecuada ventilación en ambos hemitórax. El abdomen se encontraba blando, depresible, pero con dolor a la palpación profunda en epigastrio. Peristalsis normal.

Se realiza endoscopía alta con equipo rígido en el que se aprecian tres imanes en la faringe que se extraen con pinza de caimán. Posteriormente, se efectúa panendoscopía, donde se observa esófago sin alteraciones. En estómago, a nivel del antro, se observaron ocho imanes que se extraen con red atrapa pólipos (Figura 3). Se identifica una úlcera de $4 \mathrm{~mm}$ con bordes congestivos y edematosos, en su interior se encuentra un imán incrustado (Figura 4). En el duodeno no se identifican lesiones.

Se toma nueva radiografía de abdomen observando seis imanes más (Figura 5). Por sospecha de perforación, se realiza laparotomía exploradora encontrando que el epiplón cubre al estómago y al retirarlo se observa asa de intestino delgado pegada a la curvatura mayor del estómago. Al despegar el asa se aprecia un orificio en el intestino (localizado a $30 \mathrm{~cm}$ de la válvula ileocecal), el cual comunica con otro en el estómago. En esta porción intestinal se observan los seis imanes restantes (Figura 6). Se realiza resección de $6 \mathrm{~cm}$ de íleon con anastomosis término-terminal y en estómago se reavivan

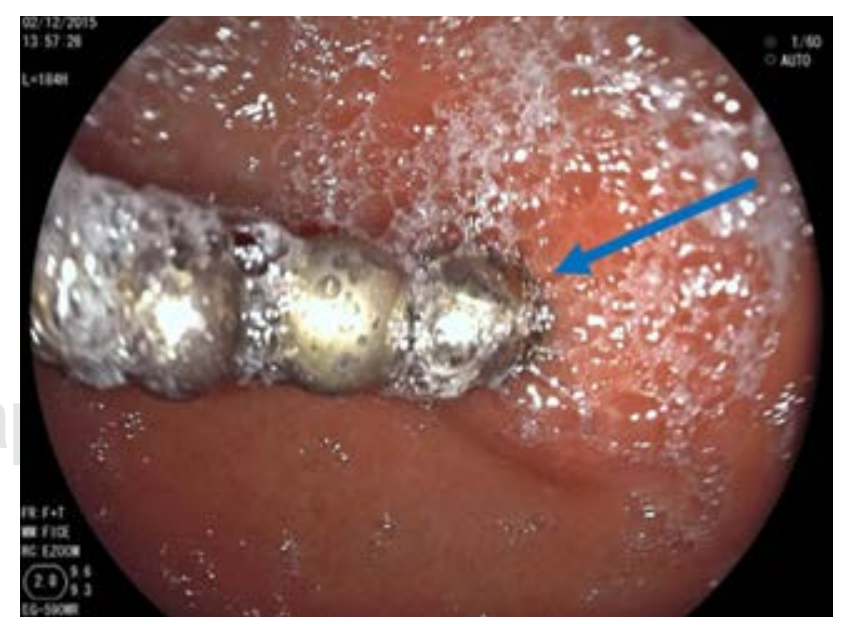

Figura 3: A través de la endoscopía en estómago se aprecian ocho imanes. 


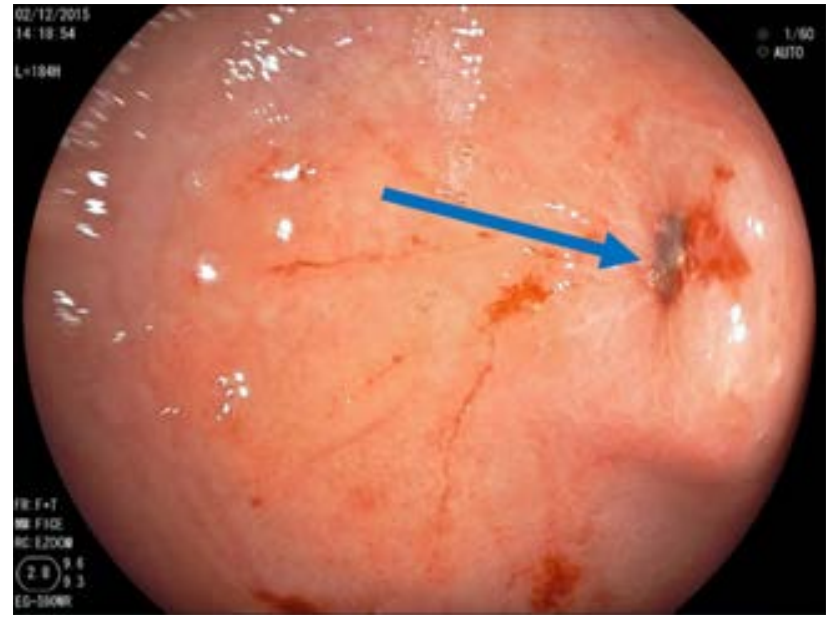

Figura 4: A través de la endoscopía se aprecia un imán incrustado en la pared del estómago.

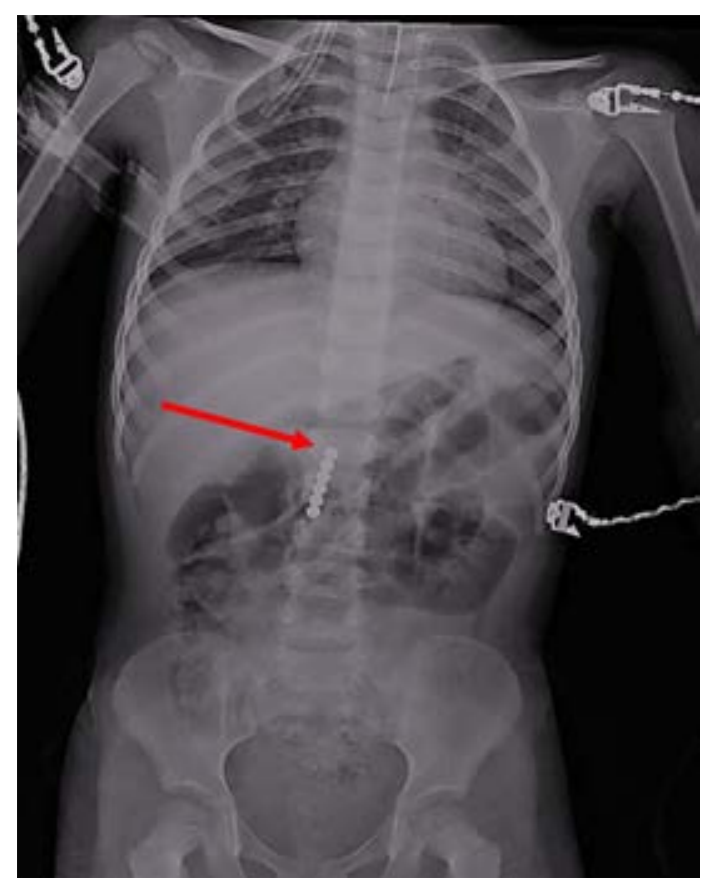

Figura 5: La radiografía muestra los seis imanes restantes en intestino.

los bordes cerrando en dos planos. Se maneja con ayuno por cinco días, sonda nasogástrica, triple esquema antibiótico, omeprazol y analgésicos. La evolución es satisfactoria y se egresa a los siete días. Se le da seguimiento por seis meses permaneciendo asintomática.

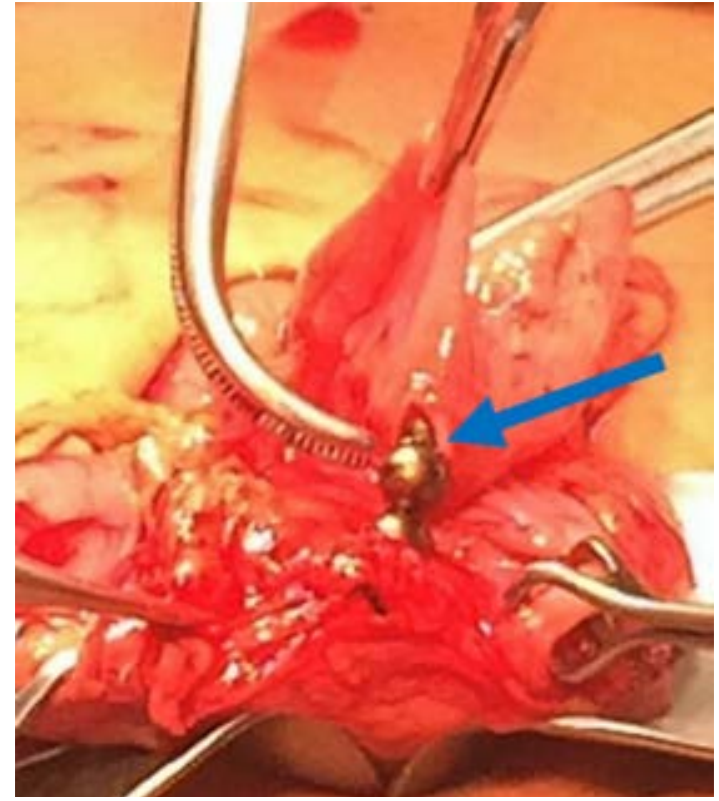

Figura 6: Extracción de los seis imanes restantes a través de laparotomía.

\section{DISCUSIÓN}

El aumento en la ingestión de pilas de botón y los imanes se debe a que se encuentran incluidos en muchos de los juguetes, lo que ha obligado a diferentes compañías a retirarlos del mercado. ${ }^{3}$ Los imanes compuestos de neodimio, boro y hierro son usados como adornos para decoración en las oficinas, ya que permiten cambiar de forma y contienen pequeñas esferas que se unen entre sí en número aproximado de 125 a $1,000{ }^{4}$

En varios casos reportados en la literatura, se refiere que el accidente fue inadvertido por los padres y se llegó al diagnóstico cuando se presentaron por un cuadro de abdomen agudo que requería cirugía o al tomar una radiografía en la que se documenta el hallazgo de los imanes. ${ }^{8-10}$ La sintomatología más frecuente observada es odinofagia, náusea, vómito, dolor abdominal o datos de irritación peritoneal, aunque hay un grupo que puede cursar asintomático. ${ }^{5,8}$ La placa simple de tórax, incluyendo cuello, y la abdominal permiten realizar el diagnóstico y visualizar si se trataba de uno o varios imanes. ${ }^{5,8}$ Kramer y colegas refieren que existen varios reportes que han documentado que los imanes pueden estar tan estrechamente unidos que parezcan uno solo, por lo que se sugiere que se tomen dos proyecciones radiográficas, para corroborar que se trate de uno o más. ${ }^{7}$ 
En cualquier caso, la recomendación es la extracción, ya sea que sea único o múltiple, si se encuentra al alcance del endoscopio, aun cuando los pacientes estén asintomáticos. Si el imán está por delante del duodeno se sugiere la vigilancia y el uso de laxantes. .,, 11 Pero si éstos ya no se encuentran al alcance del endoscopio, el seguimiento debe ser estrecho con toma seriada de radiografías abdominales y si existen datos de retención o presencia de sintomatología, se debe intervenir quirúrgicamente. ${ }^{4,7,11}$ Sola y colaboradores manejaron 60 casos en dos hospitales con ingestión de múltiples imanes; de éstos, 42 (70\%) requirieron alguna intervención que fue endoscópica (50\%), quirúrgica (31\%) y quirúrgica y endoscópica (19\%), como ocurrió en nuestro caso, los 18 restantes fueron evacuados sin problema. ${ }^{11}$

\section{REFERENCIAS}

1. Blanco-Rodríguez G, Teyssier-Morales G, Penchyna-Grub J, Madriñan-Rivas JE, Rivas-Rivera IA, Trujillo-Ponce de León A et al. Características y resultados de la ingestión de cuerpos extraños en niños. Arch Argent Pediatr. 2018; 116(4): 256-261.

2. Abbas MI, Oliva-Hemker M, Choi J, Lustik M, Gilger MA, Noel RA et al. Magnet ingestions in children presenting to US emergency departments, 2002-2011. J Pediatric Gastroenterol Nut. 2013; 57(1): 18-22.
3. Kabre R, Chin A, Rowell E, Browne M, Barsness KA, Luck S et al. Hazardous complications of multiple ingested magnets: report of four cases. Eur J Pediatric Surg. 2009; 19(3): 187-190.

4. Hussain SZ, Bousvaros A, Gilger M, Mamula P, Gupta S, Kramer $R$ et al. Management of ingested magnets in children. J Pediatric Gastroenterol Nut. 2012; 55(3): 239-242.

5. Tavarez MM, Saladino RA, Gaines BA, Manole MD. Prevalence, clinical features and management of pediatric magnetic foreign body ingestions. J Emerg Med. 2013; 44(1): 261-267.

6. Salimi A, Kooraki S, Esfahani SA, Mehdizadeh M. Multiple magnet ingestion: Is there a role for early surgical intervention? Ann Saudi Med. 2012; 32(1): 93-96.

7. Kramer RE, Lerner DG, Lin T, Manfredi M, Shah M, Stephen TC et al. Management of ingested foreign bodies in children: A clinical report of the NASPGHAN endoscopy committee. J Gastroenterol Nut. 2015; 60(4): 562-574.

8. Wildhaber BE, Le Coutre C, Genin B. Ingestion of magnets: innocent solitude harmful in groups. J Pediatric Surg. 2005; 40: E33-E35.

9. Alzahem AM, Soundappan SS, Jefferies H, Cass DT. Ingested magnets and gastrointestinal complications. J Paediatr Child Health. 2007; 43(6): 497-498.

10. Uchida K, Otake K, Iwata T, Watanabe H, Inoue M, Hatada T et al. Ingestion of multiple magnets: hazardous foreign bodies for children. Pediatr Radiol. 2006; 36(3): 263-264.

11. Sola R Jr, Rosenfeld EH, Yu YR, St Peter SD, Shah SR. Magnet foreign body ingestion: rare occurrence but big consequences. $J$ Pediatric Surg. 2018; 53(9): 1815-1819.

Conflicto de intereses: los autores declaran que no tienen. 